\title{
KNAPP-WALLACH SZEGÖ INTEGRALS. II. THE HIGHER PARABOLIC RANK CASE
}

\author{
B. E. BLANK
}

\begin{abstract}
Let $G$ be a connected reductive linear Lie group with compact center and real rank $l$. For each integer $k(1 \leqslant k \leqslant l)$ and each discrete series representation $\pi$ of $G$, an explicit embedding of $\pi$ into a generalized principal series representation induced from a parabolic subgroup of rank $k$ is given. The existence of such embeddings was proved by W. Schmid. In this paper an explicit integral formula with Szegö kernel is given which provides these mappings.
\end{abstract}

1. Introduction. To each discrete series representation $\pi_{\Lambda}$ of a connected reductive Lie group $G$ with compact center, Knapp and Wallach have associated a "Szegö kernel" which provides a $G$-equivariant map of a certain principal series representation onto $\pi_{\Lambda}$ [10 and 11]. Subsequently, the author defined analogous mappings from generalized principal series representations induced from a maximal parabolic subgroup $P_{1}$ of $G$ [2]. In this paper, under the additional assumption that $G$ has a faithful finite dimensional representation, we give such Szegö mappings for generalized principal series representations induced from standard parabolic subgroups of $G$ of any parabolic rank.

We shall describe the contents of this paper more precisely now, referring to later sections for some definitions. To simplify certain statements, we assume for this section only that $G$ is acceptable in the sense of Harish-Chandra [4]. There Harish-Chandra proved that $G$ has a discrete series $\mathscr{E}^{2}(G)$ if and only if $G$ has a Cartan subgroup $T$ contained in a maximal compact subgroup $K$ of $G$. To each nonsingular integral form $\Lambda$ on the complexification $\mathrm{t}^{\mathrm{C}}$ of the Lie algebra $\mathrm{t}$ of $T$, Harish-Chandra associates an invariant eigendistribution which is the character of a discrete series representation $\pi_{\Lambda}[3,4]$. These representations exhaust $\mathscr{E}^{2}(G)$ and two such are equivalent if and only if their parameters $\Lambda$ are conjugate under the Weyl group of $K$.

The representation $\pi_{\Lambda}$ may be realized on the space of square integrable smooth functions on $G$ that transform on one side under $K$ and that are annihilated by an appropriate first order elliptic differential operator. Differential operators such as $\partial$ and $\bar{\partial}^{*}$ and the Dirac operator have been used, but as was observed in [10, §3], Schmid's operator $\mathscr{D}_{\Lambda}$, first introduced in [15 and 16], is in some sense the most appropriate in that its kernel is contained in the kernels of the other operators listed.

Received by the editors December 10, 1984.

1980 Mathematics Subject Classification. Primary 22E46; Secondary 22E30.

(1) 1987 American Mathematical Society $0002-9947 / 87 \$ 1.00+\$ .25$ per page 
Thus, with $\pi_{\Lambda}$ so realized, the quotient mapping onto $\pi_{\Lambda}$ maps into the kernel of a well-known first order elliptic system, hence the name "Szegö kernel." Indeed, in a limiting case the Szegö kernel given by Knapp and Wallach coincides with the classical Szegö kernel for the unit ball in $\mathrm{C}^{n}[\mathbf{8}, \mathbf{1 0}]$.

Let $\alpha_{1}, \ldots, \alpha$, be a sequence of noncompact roots of $\Delta=\Delta\left(\mathrm{g}^{\mathbf{C}}, \mathrm{t}^{\mathrm{C}}\right)$ that satisfies (2.1), (2.2), and (2.3). For each $p, 1 \leqslant p \leqslant l=$ real rank of $G$, let $P_{p}$ denote the standard cuspidal parabolic subgroup with Langlands decomposition $M_{p} A_{p} N_{p}$ and parabolic rank $p$ built from $\alpha_{1}, \ldots, \alpha_{p}$. The constructions which we outline in $\S 2$ and the facts quoted concerning these parabolic subgroups are well known $[5,6, \mathbf{1 8}]$. In $\S 2$ we also discuss the finite subgroup $F$ of $K$ that controls the disconnectedness of the parabolic subgroups $P_{p}$; the properties of $F$ that we will need were obtained in [ 9 and 12]. The important point for the induction argument given in $\S 4$ is that $M_{p} \cap P_{p+1}$ is a maximal parabolic subgroup of $M_{p}$.

Let $\lambda$ be the Blattner parameter associated to the Harish-Chandra parameter $\Lambda$. Let $\left(\tau_{\lambda}, V_{\lambda}\right)$ be a $K$-type of highest weight $\lambda$. The main content of $\S 3$ is the construction of $K_{p}^{0}$-type $\left(\sigma_{\lambda}^{(p)}, H_{\lambda}^{(p)}\right)$ where $K_{p}^{0}=K \cap M_{p}^{0}, H_{\lambda}^{(p)} \subset V_{\lambda}$, and $\sigma_{\lambda}^{(p)}=$ $\tau_{\lambda} \mid K_{p}^{0}$. We also make the useful observation there that $\Lambda$ gives rise to Harish-Chandra parameters $\Lambda_{p}$ of $\mathscr{E}^{2}\left(M_{p}^{0}\right)$ by restriction.

In $\S 3$ we give a procedure for iterating the results of [2] to provide Szegö maps of the appropriate $P_{p}$-induced continuous series representations, where the parabolic rank $p$ of $P_{p}$ is arbitrary. A complication arises in the induction method we employ in that $M_{p}$ does not inherit the connectedness property of $G$ which was assumed in [2]. The simplest approach seemed to be to apply the results of [2] to $M_{p}^{0}$ and to pass to $\mathscr{E}^{2}\left(M_{p}\right)$ from $\mathscr{E}^{2}\left(M_{p}^{0}\right)$ by inducing representations of $M_{p}^{0} Z_{M_{p}}[6]$. This is facilitated by the study of the subgroup $F$ of $K[9,12]$ and its subgroups $F^{(p)}$ and $F_{p}^{(1)}$ (cf. $\left.\S \S 2,4\right)$ which control the disconnectedness of $M_{p}$ and its maximal parabolic subgroup; it was to simplify $F$ that we imposed the condition of linearity on $G$.

In [2] we showed that for the maximal parabolic $P_{1}=M_{1} A_{1} N_{1}$ of $G$ there exists a parameter $\nu_{1} \in \mathfrak{a}_{1}^{\prime}$, a character $\eta_{1}$ of $F^{(1)}$ compatible with a member $\pi_{\Lambda_{1}}$ of $\mathscr{E}^{2}\left(M_{1}^{0}\right)$, and an integral mapping $S_{1}^{G}(\Lambda)$ that carries $C^{\infty}\left(G, \pi_{\Lambda_{1}} \otimes \eta_{1} \otimes e^{\nu_{1}} \otimes 1\right)$ into the kernel of $\mathscr{D}_{\Lambda}$ in $C^{\infty}\left(G, \tau_{\lambda}\right)$. These spaces can be identified with the spaces of the generalized principal series representation $U\left(P_{1}: \pi_{\Lambda_{1}}\left(\eta_{1}\right): \nu_{1}\right)$ and discrete series representation $\pi_{\Lambda}$ respectively. The mapping is given by

$$
S_{1}^{G}(\Lambda: f)(x)=\int_{K} \tau_{\lambda}(k)^{-1} f(k x)(1) d k \quad(x \in G) .
$$

Our main result here is the specification of a member $\pi_{\Lambda_{p}}$ of $\mathscr{E}^{2}\left(M_{p}^{0}\right)$, a parameter $\nu_{p} \in \mathrm{a}_{p}^{\prime}$, and a character $\eta_{p}$ of $F^{(p)}$ for which

THEOREM 1.1. The Szegö integral defined on $C^{\infty}\left(G, \pi_{\Lambda_{p}} \otimes \eta_{p} \otimes e^{\nu_{p}} \otimes 1\right)$ by the right-hand side of (1.1) maps into the kernel of Schmid's operator $\mathscr{D}_{\Lambda}$ in $C^{\infty}\left(G, \tau_{\lambda}\right)$. Under the identification of $C^{\infty}\left(G, \pi_{\Lambda_{p}} \otimes \eta_{p} \otimes e^{\nu_{p}} \otimes 1\right)$ with the space of the generalized principal series $U\left(P_{p}: \pi_{\Lambda_{p}}\left(\eta_{p}\right): \nu_{p}\right), S_{p}^{G}(\Lambda)$ carries the $K$-finite vectors of $U\left(P_{p}: \pi_{\Lambda_{p}}\left(\eta_{p}\right): \nu_{p}\right)$ in a $\mathrm{g}$-equivariant fashion onto the $K$-finite vectors of the discrete series $\pi_{\Lambda}$. 
This theorem follows from the case $p=1$ proved in [2] and the construction in $\S 4$ of a composed map $J_{p}$ with factor $S_{1}^{\mathcal{M}_{p}^{0}}$ for which

$$
\begin{array}{cc}
C^{\infty}\left(G, \pi_{\Lambda_{p+1}} \otimes \eta_{p+1} \otimes e^{\nu_{p+1}} \otimes 1\right) & \stackrel{J_{p}}{\rightarrow} \\
S_{p+1}^{G}(\Lambda) \searrow & C^{\infty}\left(G, \pi_{\Lambda_{p}} \otimes \eta_{p} \otimes e^{\nu_{p}} \otimes 1\right) \\
& \downarrow S_{p}^{G}(\Lambda) \\
& C^{\infty}\left(G, \tau_{\lambda}\right)
\end{array}
$$

Iteration through $p=l$ recovers the Szegö maps constructed in [11], although we have not cleaned up the parameter $\nu_{l}$ as was done in $[11, \S 8]$. Nor have we discussed the case where $\Lambda$ is a limit Harish-Chandra parameter; the interested reader will have no trouble extending the statement of Theorem 1.1 to include these parameters.

The existence of such surjective maps onto discrete series is due to W. Schmid, who in a 1976 I.A.S. lecture discussed the $p=1$ case. Iteration of his character identities using induction in stages and exactness of the induction functor leads to the existence of the maps we have obtained. The virtue of the present treatment is its explicitness.

The author would like to thank Professor A. W. Knapp with whom he has had conversations concerning Szegö kernels and also the referee of [2], whose comments on that paper improved the exposition of the present one as well.

2. Standard parabolic subgroups. Throughout the remainder of this paper $G$ will be a connected reductive linear Lie group with compact center. Let $\mathfrak{g}=\mathfrak{t} \oplus \mathfrak{p}$ be a Cartan decomposition of the Lie algebra $g$ of $G$ with respect to the Cartan involution $\theta$ fixing $K$. Fix a compact Cartan subalgebra $t \subset \mathfrak{f}$ of $\mathfrak{g}$ and let $\Delta$ be the corresponding set of roots $\Delta\left(\mathrm{g}^{\mathbf{C}}, \mathrm{t}^{\mathrm{C}}\right)$. The Killing form $B$ of $\mathrm{g}^{\mathrm{C}}$ gives rise to a nondegenerate bilinear form $\langle\cdot, \cdot\rangle$ on $\left(\mathrm{t}^{\mathbf{C}}\right)^{\prime}$. A linear functional $\Lambda$ on $\mathrm{t}^{\mathbf{C}}$ is nonsingular if $\langle\Lambda, \alpha\rangle \neq 0$ for every $\alpha$ in $\Delta$. If an ordering of $\Delta$ has been given, let $\delta=\frac{1}{2} \sum_{\alpha \in \Delta^{+}} \alpha$. The discrete series of $G$ is parametrized (with repetition) by the set of nonsingular $\Lambda$ in $\left(\mathrm{t}^{\mathbf{C}}\right)^{\prime}$ for which $\Lambda-\delta$ is integral. This set does not in fact depend on the ordering that led to $\delta$ and so, given a Harish-Chandra parameter $\Lambda$, we are free to choose a convenient ordering. We do this by letting $\Delta^{+}=\{\alpha \in$ $\Delta \mid\langle\Lambda, \alpha\rangle>0\}$.

Since $\mathrm{t} \subset \mathfrak{f}$, the root spaces $\mathrm{g}_{\alpha}(\alpha \in \Delta)$ are contained either in $\mathfrak{f}^{\mathbf{C}}$ or $\mathfrak{p}^{\mathbf{C}} ; \Delta$ therefore splits into compact roots $\Delta_{k}$ and noncompact roots $\Delta_{n}$. Let $l$ be the real rank of $G$, i.e., $l=\operatorname{dim}_{\mathbf{R}} \mathfrak{a}$ where $\mathfrak{a}$ is any maximal abelian subspace of $\mathfrak{p}$. We fix a sequence $\alpha_{1}, \ldots, \alpha_{l}$ of positive noncompact roots such that

(2.1) the $\alpha_{j}$ form a strongly orthogonal set,

(2.2) for each $j=1, \ldots, l \alpha_{j}$ is a simple root in the subsystem of roots strongly orthogonal to $\alpha_{1}, \ldots, \alpha_{j-1}$,

(2.3) for each $p=1, \ldots, l$ if a compact root $\beta$ is orthogonal to $\alpha_{1}, \ldots, \alpha_{p}$ then $\beta$ is strongly orthogonal to $\alpha_{1}, \ldots, \alpha_{p}$.

The existence of such a system for any system of simple roots of $\Delta$ follows from the existence of a "fundamental sequence" [10, §4]. 
Normalize nonzero root vectors $E_{\alpha} \in \mathfrak{q}_{\alpha}$ according to [7, pp. 155-156]. Let $X_{j}=E_{\alpha_{l}}+E_{-\alpha_{l}}(j=1, \ldots, l)$. Each $X_{j}$ belongs to $\mathfrak{p}$. Form abelian subspaces $a_{p}$ of $\mathfrak{p}$ by $\mathfrak{a}_{1}=\mathbf{R} \cdot X_{1}$ and $a_{p}=a_{p-1} \oplus \mathbf{R} \cdot X_{p}(2 \leqslant p \leqslant l)$. Let $H_{j}$ be the element of $i$ t for which $\alpha_{j}(\cdot)=B\left(\cdot, H_{j}\right)$. Let $\mathfrak{h}_{p}$ be the orthogonal complement in $\mathrm{t}$ of the space spanned by $\left\{i H_{1}, \ldots, i H_{p}\right\}$ over $\mathbf{R}$. Let $\mathfrak{b}_{p}=\mathfrak{a}_{p} \oplus \mathfrak{h}_{p}$. Then $\mathfrak{b}_{p}$ is a $\boldsymbol{\theta}$-stable Cartan subalgebra of $q$ and $A_{p}=\exp a_{p}$ is the split component of a cuspidal parabolic subgroup $P_{p}$ of $G$.

Some computations with Cayley transforms [13] will be needed. Let $u_{j}=$ $\exp \frac{1}{4} \pi\left(E_{\alpha_{1}}-E_{-\alpha_{1}}\right)$ and let $c_{p}=\operatorname{Ad}\left(u_{1} \cdots u_{p}\right)$. Then $c_{p}$ carries $\mathfrak{b}_{p}^{\mathbf{C}}$ onto $\mathrm{t}^{\mathbf{C}}$ acting trivially on $\mathfrak{h}_{p}$ and taking $\mathbf{R} \cdot X_{j}$ onto $\mathbf{R} \cdot H_{j}$ for $j=1, \ldots, p$. Furthermore, ${ }^{t} c_{p}$ : $\Delta \rightarrow \Delta\left(\mathfrak{g}^{\mathbf{C}}, \mathfrak{b}_{p}^{\mathbf{C}}\right)$ carrying roots that vanish on $\mathbf{C} \cdot H_{1} \oplus \cdots \oplus \mathbf{C} \cdot H_{p}$ to roots vanishing on $\mathfrak{a}_{p}^{c}$; let $\Phi_{p}$ be the set of restrictions to $\mathfrak{h}_{p}^{\mathrm{C}}$ of such roots. If we let $\mathfrak{m}_{p}$ denote the orthogonal complement of $\mathfrak{a}_{p}$ in $Z_{\beta}\left(a_{p}\right)$, the centralizer of $a_{p}$ in $\mathfrak{g}$, then $\Phi_{p}$ is the set of roots $\Delta\left(\mathfrak{m}_{p}^{\mathbf{C}}, \mathfrak{h}_{p}^{\mathbf{C}}\right)$ of $\mathfrak{m}_{p}^{\mathbf{C}}$ with respect to $\mathfrak{h}_{p}^{\mathbf{C}}$. The ordering of $\Phi_{p}$ is transferred to $\Phi_{p}$ from $\Delta$ by ${ }^{t} c_{p}$. The following well-known Cayley transform computations for orthogonal roots $\alpha$ and $\alpha_{j}$ will be needed later:

$$
\operatorname{Ad}\left(u_{j}\right)^{-1} E_{\alpha}=E_{\alpha} \quad \text { if } \alpha \text { and } \alpha_{j} \text { are strongly orthogonal, }
$$

$$
\operatorname{Ad}\left(u_{j}\right)^{-1} E_{\alpha}=\frac{1}{2}\left(\left[E_{-\alpha}, E_{\alpha}\right]-\left[E_{\alpha,}, E_{\alpha}\right]\right) \text { otherwise. }
$$

Form $a_{p}$-roots $\Delta\left(\mathfrak{g}, a_{p}\right)(1 \leqslant p \leqslant l)$ and define an ordering on $\Delta\left(\mathfrak{g}, \mathfrak{a}_{p}\right)$ by means of the ordered basis $X_{1}, \ldots, X_{p}$ of $a_{p}$. Then the positive $a_{p}$-roots are the nonzero restrictions to $a_{p}$ of the positive $a_{\digamma}$ roots. Let $\rho_{p}$ denote half the sum of the positive $\mathfrak{a}_{p}$-roots with multiplicity. Let $\mathfrak{n}$, be the sum of the $\Delta^{+}\left(\mathfrak{g}, \mathfrak{a}_{l}\right)$-root spaces and let $N_{l}=\exp n_{l}$. Let $M_{p}^{0}$ be the analytic subgroup of $G$ corresponding to $\mathrm{m}_{p}$ and let $M_{l}=Z_{K}\left(a_{l}\right)$, the centralizer of $a_{l}$ in $K$.

We will be concerned with a family of cuspidal standard parabolic subgroups $P_{p}$ $\left(1 \leqslant p \leqslant l\right.$ ) with split components $A_{p}$. These parabolic subgroups of $G$ are obtained in a standard way $[5,18]$ from the subsets $\left\{\left.{ }^{t} c_{l} \alpha_{j}\right|_{a}: j=p+1, \ldots, l\right\}$ of simple elements of $\Delta\left(\mathfrak{g}, \mathfrak{a}_{l}\right)$. We write the Langlands decomposition of $P_{p}$ as $M_{p} A_{p} N_{p}$. Then there is a finite group $F$ defined by $F=K \cap \exp i$, with the following properties $[9,12]$ :

$$
\begin{gathered}
F \text { is generated by }\left\{\gamma_{\alpha}=\exp 2 \pi i|\alpha|^{-2} H_{\alpha}: \alpha \in \Delta\left(\mathrm{g}, \mathrm{a}_{l}\right)\right\}, \\
F \text { is a subgroup of the center } Z_{M_{l}} \text { of } M_{l}, \text { and } \\
M_{p}=M_{p}^{0} F .
\end{gathered}
$$

We have the inclusion relations $P_{1} \supset \cdots \supset P_{l}[18$, p. 283]; the maximal and minimal parabolics $P_{1}$ and $P_{l}$ are noteworthy, the former for the facility of dealing with the one dimensional $A_{1}$, the latter for the compactness of $M_{l}$. Let $a^{p}=$ $\sum_{j=p+1}^{l} \mathbf{R} \cdot X_{j}$ for $p=1, \ldots, l-1$. Then $a^{p}$ plays the same role for $M_{p}$ as $a_{l}$ does for $G$. If $A^{p}$ is the analytic subgroup of $G$ corresponding to $a^{p}$ then $A_{p} A^{p}=A_{\text {l }}$ and if $N^{p}=M_{p} \cap N_{l}$ then $N_{p} N^{p}=N_{l}$ and $M_{l} A^{p} N^{p}$ is a minimal parabolic subgroup of $M_{p}$. Now $\left.\alpha_{p+1}\right|_{\mathfrak{h} \mathrm{h}_{p}^{\mathrm{C}}}=\left.\left({ }^{t} c_{p} \alpha_{p+1}\right)\right|_{\mathfrak{h} p}$ belongs to $\left(\Phi_{p}\right)_{n}$ and its root vector in $\mathfrak{m}_{p}$ is $E_{\alpha_{p}, 1}$ by (2.4). Thus, if $\tilde{\mathfrak{a}}_{p+1}=\mathbf{R} \cdot X_{p+1}$, then $\tilde{A}_{p+1}=\exp \tilde{\mathfrak{a}}_{p+1}$ serves the 
same role in $M_{p}$ as $A_{1}$ does in $G$ and $A_{p+1}=A_{p} \tilde{A}_{p+1}$. Then $Z_{\mathrm{m}_{p}}\left(X_{p+1}\right)-\mathbf{R} \cdot X_{p+1}$, which is nothing but $\mathfrak{m}_{p+1}$, plays the same role in $\mathfrak{m}_{p}$ as $M_{1}$ does in $\mathrm{g}$. Since $M_{l}$ has the same role in both $M_{p}$ and $G, M_{p+1}=M_{p+1}^{0} M_{l}$ arises in $M_{p}$ in the same way it does in $G$ and $M_{p+1} \tilde{A}_{p+1}$ is the reductive component of a maximal parabolic subgroup of $M_{p}$. The $\tilde{a}_{p+1}$-roots with respect to $\mathfrak{m}_{p}$ are the restrictions to $\tilde{a}_{p+1}$ of $\mathfrak{a}_{p+1}$-roots (with respect to $\mathfrak{g}$ ) that vanish on $\mathfrak{a}_{p}$. Let $\tilde{\rho}_{p+1}$ be half the sum of the positive elements in $\Delta\left(\mathfrak{m}_{p}, \tilde{\mathfrak{a}}_{p+1}\right)$ with multiplicity. By [18, p. 290], if $\tilde{N}_{p+1}$ is the nilpotent Langlands component of this maximal parabolic subgroup of $M_{p}$, then $N_{p+1}=N_{p} \tilde{N}_{p+1}$ and $\tilde{N}_{p+1}=M_{p} \cap N_{p+1}$.

In the preceding paragraph we have arrived at a maximal parabolic subgroup $M_{p+1} \tilde{A}_{p+1} \tilde{N}_{p+1}$ of $M_{p}$. However, it is the corresponding maximal parabolic subgroup of $M_{p}^{0}$ that we shall require. In this situation, the finite group that controls the disconnectedness of the reductive component of the maximal parabolic subgroup of $M_{p}^{0}$ is $F_{p}=K_{p}^{0} \cap \exp i a_{\text {, where }} K_{p}^{0}=K \cap M_{p}^{0}$ is the maximal compact subgroup of $M_{p}^{0}$. As the notation indicates, $K_{p}^{0}$ is the identity component of $K_{p}=K \cap M_{p}$, the maximal compact subgroup of $M_{p}$. Note that $F_{p}=F \cap M_{p}^{0}$. The results quoted earlier show that $\left(M_{p+1}^{0} F_{p}\right) \tilde{A}_{p+1} \tilde{N}_{p+1}$ is a maximal parabolic subgroup of $M_{p}^{0}$.

3. Harish-Chandra and Blattner parameters for $M_{p}^{0}$. Let $\Lambda \in\left(\mathrm{t}^{\mathrm{C}}\right)^{\prime}$ be a HarishChandra parameter for a discrete series representation $\pi_{\Lambda}$ of $G$. Order $\Delta=\Delta\left(\mathrm{g}^{\mathrm{C}}, \mathrm{t}^{\mathrm{C}}\right)$ so that $\Lambda$ is dominant. Let $\delta, \delta_{k}$, and $\delta_{n}$ be half the sum of the elements of $\Delta^{+}, \Delta_{k}^{+}$, and $\Delta_{n}^{+}$respectively. Then $\lambda=\Lambda-\delta_{k}+\delta_{n}$ is the Blattner parameter associated to $\Lambda$ and $\lambda$ is integral, $\Delta_{k}^{+}$-dominant and the lowest $K$-type in $\pi_{\Lambda}$. Let $\left(\tau_{\lambda}, V_{\lambda}\right)$ be an irreducible representation of $K$ on $V_{\lambda}$ with nonzero highest weight $\phi_{\lambda}$.

For each $p=1, \ldots, l-1$ let $\Lambda_{p}=\left.\Lambda\right|_{\mathfrak{h}_{p}^{\text {c. }}}$. Since $\mathfrak{h}_{p}$ is a compact Cartan subalgebra of $\mathfrak{m}_{p}$, the discrete series $\mathscr{E}^{2}\left(M_{p}^{0}\right)$ of $M_{p}^{0}$ is indexed by Harish-Chandra parameters on $\mathfrak{h}_{p}^{\mathrm{C}}$. Let $\delta^{(p)}, \delta_{k}^{(p)}$, and $\delta_{n}^{(p)}$ be half the sum of the elements of $\Phi_{p}^{+}$, $\left(\Phi_{p}\right)_{k}^{+}$, and $\left(\Phi_{p}\right)_{n}^{+}$respectively. Let $H_{\lambda}^{(p)}$ be the $K_{p}^{0}$-cyclic subspace of $V_{\lambda}$ generated by $\phi_{\lambda}$ under $\tau_{\lambda}$. Denote by $\sigma_{\lambda}^{(p)}$ the restriction of $\left.\tau_{\lambda}\right|_{K_{p}^{0}}$ to $H_{\lambda}^{(p)}$.

Proposition 3.1. The restriction of $\lambda$ to $\mathfrak{h}_{p}^{\mathbf{C}}$ is an integral form on $\mathfrak{h}_{p}^{\mathbf{C}}$ dominant with respect to $\left(\Phi_{p}\right)_{k}^{+}$. Indeed, $\left(\sigma_{\lambda}^{(p)}, H_{\lambda}^{(p)}\right)$ is an irreducible representation of $K_{p}^{0}$.

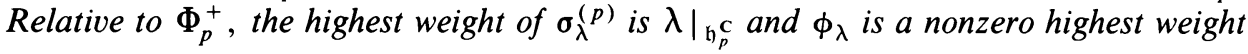
vector.

Proof. The argument runs along the lines of [10, Proposition 5.5] which treats the $p=l$ case with $M_{l}$ rather than $M_{l}^{0}$. The result stated there needed correction because of the disconnectedness of $M_{l}$ (cf. [11]). We avoid this complication by using $K_{p}^{0}$.

To prove that $\phi_{\lambda}$ is a highest weight vector for $\sigma_{\lambda}^{(p)}$ we are to show that $\sigma_{\lambda}^{(p)}\left(c_{p} E_{\beta}\right)=0$ if $\beta \in \Delta^{+}$with $\left\langle\beta, \alpha_{j}\right\rangle=0$ for $1 \leqslant j \leqslant p$ and with $c_{p} E_{\beta} \in \mathrm{t}^{\mathrm{C}}$. If $\beta$ satisfies these properties and is a compact root, then by (2.3) and (2.4), $c_{p} E_{\beta}=E_{\beta}$ and $\sigma_{\lambda}^{(p)}\left(c_{p} E_{\beta}\right) \phi_{\lambda}=\tau_{\lambda}\left(E_{\beta}\right) \phi_{\lambda}=0$ since $\phi_{\lambda}$ is a highest weight vector for $\tau_{\lambda}$.

Next, let $\beta$ be noncompact. If $\beta$ were strongly orthogonal to each $\alpha_{j}, j=1, \ldots, p$, then we would have $c_{p} E_{\beta}=E_{\beta} \in \mathfrak{p}^{\mathbf{C}}$, so we may suppose that $\beta$ is not strongly orthogonal to some $\alpha_{j}$ for some $j<p+1$. Then, by (2.5), there is a constant $a$ for 
which $\operatorname{Ad}\left(u_{j}\right) E_{\beta}=a E_{\beta+\alpha_{j}}-a E_{\beta-\alpha_{j}}$. For $1 \leqslant i \leqslant p, i \neq j, \alpha_{i} \pm\left(\beta \pm \alpha_{j}\right)$ cannot be roots since then we would have roots of three different lengths. Thus, all other $\operatorname{Ad}\left(u_{i}\right)$ fix $\operatorname{Ad}\left(u_{j}\right) E_{\beta}$ and we obtain $c_{p} E_{\beta}=a E_{\beta+\alpha_{i}}-a E_{\beta-\alpha_{j}}$. Since this argument also shows that $\beta$ is strongly orthogonal to $\alpha_{1}, \ldots, \alpha_{j-1}$ and since $\alpha_{j}$ is simple in such a subsystem of roots by (2.2), we must have $\beta-\alpha_{j}>0$. Thus $\beta \pm \alpha_{j}$ belong to $\Delta_{k}^{+}$and $\tau_{\lambda}\left(E_{\beta \pm \alpha_{i}}\right) \phi_{\lambda}=0$.

Let $\lambda_{p} \in\left(\mathfrak{h}_{p}^{\mathbf{C}}\right)^{\prime}$ be defined by $\lambda_{p}=\Lambda_{p}-\delta_{k}^{(p)}+\delta_{n}^{(p)}$.

Proposition 3.2. The linear functional $\Lambda_{p}$ on $\mathfrak{h}_{p}^{\mathbf{C}}$ is a Harish-Chandra parameter for $M_{p}^{0}$. Furthermore, the Blattner parameter $\lambda_{p}$ associated to $\Lambda_{p}$ is the restriction to $\mathfrak{h}_{p}^{\mathrm{C}}$ of the Blattner parameter $\lambda$ associated to $\Lambda$.

Proof. The main point here is that

$$
\delta_{k}^{(p)}-\delta_{n}^{(p)}=\left.\left(\delta_{k}-\delta_{n}\right)\right|_{\mathfrak{h})_{p}^{C}}
$$

This is done first for the maximal parabolic case $p=1$ and then by induction for the general case using the method outlined in $\$ 2$ in which $m_{p+1}$ is seen to have the same role in $m_{p}$ as $m_{1}$ in $g$ (cf. $\left.[17, \S 7]\right)$. The last assertion that $\lambda_{p}=\left.\lambda\right|_{\mathfrak{h}_{p}}$ is an immediate consequence of this. It follows from Proposition 3.1 that $\lambda_{p}$ is integral and therefore $\Lambda_{p}-\delta^{(p)}$ is integral as well.

Suppose $\alpha^{\prime}=\left.{ }^{t} c_{p} \alpha\right|_{\mathfrak{h}_{p}^{\mathrm{c}}}$ belongs to $\Phi_{p}$ where $\alpha \in \Delta$ with $\left\langle\alpha, \alpha_{j}\right\rangle=0(1 \leqslant j \leqslant p)$. If $\alpha$ is strongly orthogonal to each $\alpha_{j}, 1 \leqslant j \leqslant p$, then $E_{\alpha^{\prime}}$ (the root vector in $\mathrm{m}_{p}^{\mathrm{C}}$ ) is $E_{\alpha}$ by (2.4) and so the vector $H_{\alpha^{\prime}}$ representing $\alpha^{\prime}$ by the Killing form $B_{p}$ of $\mathrm{m}_{p}^{\mathrm{C}}$ is $H_{\alpha}$. In this case $\Lambda_{p}\left(H_{\alpha^{\prime}}\right)=\Lambda\left(H_{\alpha}\right) \neq 0$ since $\Lambda$ is a Harish-Chandra parameter for $G$. If $\alpha$ fails to be strongly orthogonal to some $\alpha_{j}(1 \leqslant j \leqslant p)$, then the proof of Proposition 3.1 shows this $j$ to be unique and $E_{\alpha^{\prime}}=c_{p} E_{\alpha}=a E_{\alpha+\alpha_{j}}-a E_{\alpha-\alpha_{j}}$. Then

$$
H_{\alpha^{\prime}}=b\left(H_{\alpha+\alpha,}+H_{\alpha-\alpha_{j}}\right)
$$

and

$$
\Lambda_{p}\left(H_{\alpha^{\prime}}\right)=b \Lambda\left(H_{\alpha+\alpha_{j}}+H_{\alpha-\alpha_{j}}\right)=2 b \Lambda\left(H_{\alpha}\right) \neq 0 .
$$

It is worth noting that Proposition 3.2 remains valid if $\Lambda$ is allowed to be orthogonal to any subset of $\left\{\alpha_{1}, \ldots, \alpha_{p}\right\}$ as is evident from the proof. Another obvious but useful observation is that

$$
K_{p+1}^{0}=K_{p}^{0} \cap\left(M_{p+1}^{0} F_{p}\right)^{0}
$$

thus, if we start with the $K_{p}^{0}$-type $\left(\sigma_{\lambda}^{(p)}, H_{\lambda}^{(p)}\right)$ and the maximal parabolic subgroup $\left(M_{p+1}^{0} F_{p}\right) \tilde{A}_{p+1} \tilde{N}_{p+1}$ of $M_{p}^{0}$, the procedure analogous to going from $\left(\tau_{\lambda}, V_{\lambda}\right)$ to $\left(\sigma_{\lambda}^{(1)}, H_{\lambda}^{(1)}\right)$ gives rise to the $K_{p+1}^{0}$-type $\left(\sigma_{\lambda}^{(p+1)}, H_{\lambda}^{(p+1)}\right)$.

4. Szegö integrals. It will be convenient to have the following notation: if $X$ is a subgroup of $Y$ and $\left(\xi, H^{\xi}\right)$ is a representation of $X$ on $H^{\xi}$, it will be convenient to define

$$
C^{\infty}(Y, \xi)=\left\{f \in C^{\infty}\left(Y, H^{\xi}\right): f(x y)=\xi(x) f(y), x \in X, y \in Y\right\} .
$$


Let $\Lambda \in\left({ }^{C}\right)^{\prime}$ be a Harish-Chandra parameter for the discrete series representation $\pi_{\Lambda}$ of $G$. Let $\pi_{\Lambda_{p}}$ denote the discrete series representation of $M_{p}^{0}$ associated to $\Lambda_{p}(1 \leqslant p \leqslant l-1)$. The representation space of $\pi_{\Lambda}$ (respectively $\pi_{\Lambda_{p}}$ ) can be taken to be the space of square integrable elements of $C^{\infty}\left(G, \tau_{\lambda}\right)$ (respectively $\left.C^{\infty}\left(M_{p}^{0}, \sigma_{\lambda}^{(p)}\right)\right)$ that are annihilated by Schmid's operator $\mathscr{D}_{\Lambda}$ (respectively $\mathscr{D}_{\Lambda_{p}}$ ). Discrete series representations of $M_{p}$ are obtained by inducing to $M_{p}$ representations $\pi_{\Lambda_{p}} \otimes \eta$ of $M_{p}^{0} Z_{M_{p}}$ where $\eta$ is a character of the center $Z_{M_{p}}$ of $M_{p}$ that is compatible with $\pi_{\Lambda_{p}}[4,9]$. In $[12, \S 2]$ it is shown that the subgroup $F^{(p)}$ of $Z_{M_{p}} \cap F$ generated by $\left\{\gamma_{\alpha}\left|\alpha \in{ }^{t} c_{p} \Delta=\Delta\left(\mathfrak{g}^{\mathbf{C}}, \mathfrak{b}_{p}^{\mathbf{C}}\right), \alpha\right|_{\mathfrak{h}_{p}}=0\right\}$ satisfies $M_{p}^{0} Z_{M_{p}}=M_{p}^{0} F^{(p)}$ and we need only take $\eta$ to be a character on $F^{(p)}$ compatible with $\pi_{\Lambda_{p}}$. We let

$$
\pi_{\Lambda_{p}}(\eta)=\underset{M_{p}^{0} F^{(p)} \uparrow M_{p}}{\text { Ind }}\left(\pi_{\Lambda_{p}} \otimes \eta\right)
$$

be the resulting member of $\mathscr{E}^{2}\left(M_{p}\right)$.

We will use the (consistent) notation $G=M_{0}^{0}$. We will be concerned with generalized principal series representations of $M_{0}^{0}, M_{1}^{0}, \ldots, M_{l-1}^{0}$. If $Q=M_{Q} A_{Q} N_{Q}$ is the Langlands decomposition of a cuspidal parabolic subgroup of $M_{p}^{0}$, we will let $U_{p}\left(Q: \pi_{Q}: \nu_{Q}\right)$ be the generalized principal series representation of $M_{p}^{0}$ induced from $\pi_{Q} \otimes e^{\nu_{Q}} \otimes 1$ on $Q$, where $\pi_{Q} \in \mathscr{E}^{2}\left(M_{Q}\right)$ and $\nu_{Q} \in\left(\mathfrak{a}_{Q}^{\prime}\right)^{\mathrm{C}}$. A dense subspace on which $U_{p}\left(Q ; \pi_{Q}: \nu_{Q}\right)$ acts is $C^{\infty}\left(M_{p}^{0}, \pi_{Q} \otimes e^{\left.\nu_{Q} \otimes 1\right)}\right.$. For an element $f$ of this space we have

$$
U_{p}\left(Q: \pi_{Q}: v_{Q}: m\right) f(x)=f(x m) \quad\left(x, m \in M_{p}^{0}\right) .
$$

When $p=0$ we will drop the subscript. By Mackey's “induction in stages" theorem [14, p. 109], $U\left(P_{p}: \pi_{\Lambda_{p}}(\eta): \nu\right)$ is equivalent to

$$
\mathscr{U}\left(\pi_{\Lambda_{p}}: \eta: \nu\right)=\operatorname{Ind}_{M_{p}^{0} F^{(p)} A_{p} N_{p} \uparrow G}\left(\pi_{\Lambda_{p}} \otimes \eta \otimes e^{\nu} \otimes 1\right)
$$

A dense subspace is $C^{\infty}\left(G, \pi_{\Lambda_{p}} \otimes \eta \otimes e^{\nu} \otimes 1\right)$. The analogous representation of $M_{p}^{0}$ will be denoted by $\mathscr{U}_{p}$.

Our inductive hypothesis is that there exists a parameter $\nu_{p}=\nu_{p}(\Lambda)$ in $a_{p}^{\prime}$, a character $\eta_{p}$ of $F^{(p)}$ compatible with $\pi_{\Lambda_{p}}$, and an integral mapping $S_{p}^{G}(\Lambda)$ that carries $\mathscr{U}\left(\pi_{\Lambda_{p}}: \eta_{p}: \nu_{p}\right)$ onto $\pi_{\Lambda}$ in a $G$-equivariant fashion.

The validity of this hypothesis for $p=1$ is the subject of [2]. The parameter $\nu_{1}$ is defined by

$$
\nu_{1}\left(X_{1}\right)=\rho_{1}\left(X_{1}\right)-2\left\langle\Lambda, \alpha_{1}\right\rangle /\left|\alpha_{1}\right|^{2} .
$$

Since $F^{(1)}=\left\{1, \gamma_{\alpha_{1}}\right\}$,

$$
\eta_{1}\left(\gamma_{\alpha_{1}}\right)=(-1)^{2\left|\alpha_{1}\right|^{-2\left\langle\alpha_{1}, \lambda\right\rangle}}
$$

determines $\eta_{1}$ on $F^{(1)}$. For $f$ in $C^{\infty}\left(G, \pi_{\Lambda_{1}} \otimes \eta_{1} \otimes e^{\nu_{1}} \otimes 1\right)$, the Szegö map is given by

$$
S_{1}^{G}(\Lambda: f: x)=\int_{K} \tau_{\lambda}(k)^{-1} f(k x)(1) d k \quad(x \in G) .
$$


Note that elements of $C^{\infty}\left(G, \pi_{\Lambda_{1}} \otimes \eta_{1} \otimes e^{\nu_{1}} \otimes 1\right)$ have values in the representation space of $\pi_{\Lambda_{1}}$, which is taken to be the subspace of $C^{\infty}\left(M_{1}^{0}, \sigma_{\lambda}^{(1)}\right)$ described earlier. Thus, evaluation of $f(k x)(\cdot)$ in (4.3) is at the identity element of $M_{1}^{0}$. Then $f(k x)(1)$ takes values in $H_{\lambda}^{(1)}$ and since $H_{\lambda}^{(1)} \subset V_{\lambda},(4.3)$ is well defined. It is clear that $S_{1}^{G}(\Lambda)$ is $G$-equivariant and $S_{1}^{G}(\Lambda: f)$ belongs to $C^{\infty}\left(G, \tau_{\lambda}\right)$. The reader may consult [2] for the proof that $\mathscr{D}_{\Lambda} S_{1}^{G}(\Lambda: f)=0$ and the identification of the image of $S_{1}^{G}(\Lambda)$ with $\pi_{\Lambda}$.

We apply this result to the case where the group $G$ is taken to be $M_{p}^{0}$ and the maximal parabolic subgroup of $M_{p}^{0}$ is $\left(M_{p+1}^{0} F_{p}\right) \tilde{A}_{p+1} \tilde{N}_{p+1}$. Table 1 contains the relevant objects pertaining to $G$ and the corresponding objects of $M_{p}^{0}$.

TABLE 1

\begin{tabular}{l|l}
\multicolumn{1}{c|}{$G$} & \multicolumn{1}{c}{$M_{p}^{0}$} \\
\hline$M_{1}^{0} F^{(1)} A_{1} N_{1}$ & $M_{p+1}^{0} F_{p}^{(1)} \tilde{A}_{p+1} \tilde{N}_{p+1}$ \\
$\rho_{1}$ & $\tilde{\rho}_{p+1}=\frac{1}{2} \sum_{\alpha \in \Delta^{+}\left(\mathrm{m}_{p}, \tilde{\mathrm{a}}_{p+1}\right)}^{\alpha}$ \\
$B ;\langle\cdot, \cdot\rangle$ & $B_{p} ;\langle\cdot \cdot\rangle_{p}$ \\
$\Lambda$ & $\Lambda_{p}$ \\
$\Lambda_{p}$ & $\Lambda_{p+1}$ \\
$\left(\tau_{\lambda}, V_{\lambda}\right) ;\left(\sigma_{\lambda}^{(1)}, H_{\lambda}^{(1)}\right)$ & $\left(\sigma_{\lambda}^{(p)}, H_{\lambda}^{(p)}\right) ;\left(\sigma_{\lambda}^{(p+1)}, H_{\lambda}^{(p+1)}\right)$ \\
$\gamma_{\alpha_{1}} ; \eta_{1}$ & $\gamma_{\alpha_{p+1}} ; \tilde{\eta}_{p+1}$
\end{tabular}

In Table $1 F_{p}^{(1)}=\left\{1, \gamma_{\alpha_{p+1}}\right\}$ is the subgroup of $Z_{M_{p+1}^{0} F_{p}} \cap F_{p}$ which plays the same role in $M_{p}^{0}$ that the subgroup $F^{(1)}=\left\{1, \gamma_{\alpha_{1}}\right\}$ of $Z_{M_{1}} \cap \stackrel{M_{p+1}}{F}$ plays in $G$. With the listed conversions, $\tilde{\nu}_{p+1}$ in $\tilde{a}_{p+1}^{\prime}$ is defined by (4.2) and $\tilde{\eta}_{p+1}\left(\gamma_{\alpha_{p+1}}\right)$ by (4.3). For $f$ in the dense subspace $C^{\infty}\left(M_{p}^{0}, \pi_{\Lambda_{p+1}} \otimes \tilde{\eta}_{p+1} \otimes e^{\tilde{v}_{p+1}} \otimes 1\right)$ of $\mathscr{\mathcal { U }}_{p}\left(\pi_{\Lambda_{p+1}}: \tilde{\eta}_{p+1}: \tilde{v}_{p+1}\right)$ the Szegö map $S_{1}^{M_{p}^{0}}\left(\Lambda_{p}: f\right)$ is given by

$$
S_{1}^{M_{p}^{0}}\left(\Lambda_{p}: f: m\right)=\int_{K_{p}^{0}} \sigma_{\lambda}^{(p)}\left(k_{p}\right)^{-1} f\left(k_{p} m\right)(1) d k_{p} \quad\left(m \in M_{p}^{0}\right) .
$$

The image of $S_{1}^{M_{p}^{0}}\left(\Lambda_{p}\right)$ is $\pi_{\Lambda_{p}}$ realized in $C^{\infty}\left(M_{p}^{0}, \sigma_{\lambda}^{(p)}\right)$.

The definition of $S_{p+1}^{G}(\Lambda)$ can now be easily given. We define the parameter $\nu_{p+1}$ on $\mathfrak{a}_{p+1}=\mathfrak{a}_{p} \oplus \tilde{\mathfrak{a}}_{p+1}$ by

$$
\nu_{p+1}=\nu_{p}+\tilde{\nu}_{p+1}
$$

where each parameter $\nu_{p}$ and $\tilde{\nu}_{p+1}$ is extended by zero on the complementary subspace of $a_{p+1}$. The finite group $F^{(p+1)}$ is given by

$$
\begin{aligned}
F^{(p+1)} & =\operatorname{span}\left\{\gamma_{\alpha}: \alpha \in \Delta, \alpha\left(\mathfrak{h}_{p+1}^{\mathbf{C}}\right)=0\right\} \\
& =\operatorname{span}\left\{\gamma_{\alpha}: \alpha \in \Delta, H_{\alpha} \in \mathbf{C} H_{\alpha_{1}} \oplus \cdots \oplus \mathbf{C} H_{\alpha_{p+1}}\right\} .
\end{aligned}
$$

The inductive definition of $\eta_{p}$ on $F^{(p)}$ and the definition of $\tilde{\eta}_{p+1}$ on $F_{p}^{(1)}=$ $\left\{1, \gamma_{\alpha_{p+1}}\right\}$ therefore determine the character $\eta_{p+1}$ on $F^{(p+1)}$. The discrete series parameter on $M_{p+1}^{0}$ is taken to be $\pi_{\Lambda_{p+1}}$. We are therefore to provide a $G$-equivariant map from the representation space

$$
C^{\infty}\left(G, \pi_{\Lambda_{p+1}} \otimes \eta_{p+1} \otimes e^{\nu_{p+1}} \otimes 1\right)
$$


of $\mathscr{U}\left(\pi_{\Lambda_{p+1}}: \eta_{p+1}: \nu_{p+1}\right)$ onto $\pi_{\Lambda}$. We claim that for $f$ in

$$
C^{\infty}\left(G, \pi_{\Lambda_{p+1}} \otimes \eta_{p+1} \otimes e^{\nu_{p+1}} \otimes 1\right),
$$

the map is

$$
S_{p+1}^{G}(\Lambda: f: x)=\int_{K} \tau_{\lambda}(k)^{-1} f(k x)(1) d k \quad(x \in G) .
$$

That this formula has meaning follows from the discussion that showed (4.4) has meaning. Once again it is clear that $S_{p+1}^{G}(\Lambda)$ is $G$-equivariant and has image in $C^{\infty}\left(G, \tau_{\lambda}\right)$. That its image is nonzero can be shown by constructing an element, analogous to the one given in [2], that is not mapped to zero. Simpler and preferable in that it uses nothing about $S_{l}^{G}$ other than that it is nonzero, which is very easily proved [11], is to observe from Proposition 4.1 that if $S_{p}^{G}(\Lambda)$ were zero for any $p$, iteration would lead to $S_{l}^{G}(\Lambda)$ being zero. Indeed, the next proposition shows that $\pi_{\Lambda}$ is the image of $S_{p+1}^{G}(\Lambda)$.

Define $j_{p}$ on $G \times C^{\infty}\left(G, \pi_{\Lambda_{p+1}} \otimes \eta_{p+1} \otimes e^{\nu_{p+1}} \otimes 1\right)$ by

$$
j_{p}(x: f)=\left.\left(\mathscr{U}\left(\pi_{\Lambda_{p+1}}: \eta_{p+1}: \nu_{p+1}: x\right) f\right)\right|_{M_{p}^{0}},
$$

i.e., for $m$ in $M_{p}^{0} j_{p}(x: f)(m)=f(m x)$. Assuming for the moment that $j_{p}(x: f)$ is in the dense subspace $C^{\infty}\left(M_{p}^{0}, \pi_{\Lambda_{p+1}} \otimes \tilde{\eta}_{p+1} \otimes e^{\tilde{p}_{p+1}} \otimes 1\right)$ of $\mathscr{U}_{p}\left(\pi_{\Lambda_{p+1}}: \tilde{\eta}_{p+1}: \tilde{v}_{p+1}\right)$ on which $S_{1}^{M_{p}^{0}}\left(\Lambda_{p}\right)$ is defined, let $J_{p} f$ be defined on $G$ by

$$
J_{p} f(x)=S_{1}^{M_{p}^{0}}\left(\Lambda_{p}: j_{p}(x: f)\right) .
$$

PROPOSITION 4.1. Let $x$ belong to $G$ and $f$ to $C^{\infty}\left(G, \pi_{\Lambda_{p+1}} \otimes \eta_{p+1} \otimes e^{\nu_{p+1}} \otimes 1\right)$. The map $j_{p}$ defined by (4.8) takes values in $C^{\infty}\left(M_{p}^{0}, \pi_{\Lambda_{p+1}} \otimes \tilde{\eta}_{p+1} \otimes e^{\tilde{p}_{p+1}} \otimes 1\right)$ and so the map $J_{p} f$ is well defined with values in $\pi_{\Lambda_{p}}$. Furthermore, $J_{p} f$ belongs to $C^{\infty}\left(G, \pi_{\Lambda_{p}} \otimes \eta_{p} \otimes e^{\nu_{p}} \otimes 1\right)$ and

$$
S_{p+1}^{G}(\Lambda: f)=S_{p}^{G}\left(\Lambda: J_{p} f\right) .
$$

PROOF. It clearly suffices to prove the first assertion when $x=1$, that is, to show that $j_{p}(1: f)=\left.f\right|_{M_{p}^{0}}$ belongs to $C^{\infty}\left(M_{p}^{0}: \pi_{\Lambda_{p+1}} \otimes \tilde{\eta}_{p+1} \otimes e^{\tilde{z}_{p+1}} \otimes 1\right)$. That $\left.f\right|_{M_{p}^{0}}$ satisfies this transformation law by elements of $M_{p+1}^{0} F_{p}^{(1)} \tilde{A}_{p+1} \tilde{N}_{p+1}$ follows immediately from the transformation law of $f$ by elements of $M_{p+1}^{0} F^{(p+1)} A_{p+1} N_{p+1}$.

Next observe that for $m^{\prime}$ in $M_{p}^{0}$ we have

$$
J_{p} f(x)\left(m^{\prime}\right)=\int_{K_{p}^{0}} \sigma_{\lambda}^{(p)}\left(k_{p}\right)^{-1} f\left(k_{p} m^{\prime} x\right)(1) d k_{p} .
$$

Now let $m, z, a$, and $n$ belong to $M_{p}^{0}, F^{(p)}, A_{p}$, and $N_{p}$ respectively. Since $F^{(p)}$ is contained in both $Z_{M_{p}}$ and $K$, since $\eta_{p}$ agrees with the scalar action of $\left.\sigma_{\lambda}^{(p)}\right|_{F^{(p)}}$, since the integration in (4.11) is over elements that centralize $A_{p}$ and normalize $N_{p}$, 
and since $f$ transforms as it does, we have

$$
\begin{aligned}
J_{p} f(\operatorname{mzanx})\left(m^{\prime}\right) & =\int_{K_{p}^{0}} \sigma_{\lambda}^{(p)}\left(k_{p}\right)^{-1} f\left(k_{p} m^{\prime} m z a n x\right)(1) d k_{p} \\
& =e^{\nu_{p} \log a} \int_{K_{p}^{0}} \sigma_{\lambda}^{(p)}\left(k_{p}\right)^{-1} f\left(k_{p} z m^{\prime} m x\right)(1) d k_{p} \\
& =e^{\nu_{p} \log a} \sigma_{\lambda}^{(p)}(z) \int_{K_{p}^{0}} \sigma_{\lambda}^{(p)}\left(k_{p}\right)^{-1} f\left(k_{p} m^{\prime} m \cdot x\right)(1) d k_{p} \\
& =e^{\nu_{p} \log a} \eta_{p}(z) J_{p}(x)\left(m^{\prime} m\right) \\
& =e^{\nu_{p} \log a} \eta_{p}(z)\left(\pi_{\Lambda_{p}}(m) J_{p}(x)\right)\left(m^{\prime}\right) .
\end{aligned}
$$

Thus $J_{p} f$ belongs to $C^{\infty}\left(G, \pi_{\Lambda_{p}} \otimes \eta_{p} \otimes e^{\nu_{p}} \otimes 1\right)$.

Finally,

$$
\begin{aligned}
S_{p}^{G}\left(\Lambda: J_{p} f\right)(x) & =\int_{K} \tau_{\lambda}(k)^{-1} J_{p} f(k x)(1) d k \\
& =\int_{K} \tau_{\lambda}(k)^{-1} \int_{K_{p}^{0}} \sigma_{\lambda}^{(p)}\left(k_{p}\right)^{-1} f\left(k_{p} k x\right)(1) d k_{p} d k \\
& =\int_{K} \tau_{\lambda}(k)^{-1} f(k x)(1) d k=S_{p+1}^{G}(\Lambda: f)(x)
\end{aligned}
$$

\section{REFERENCES}

1. B. E. Blank, Embedding limits of discrete series of semisimple Lie groups, Canad. Math. Soc. Conf. Proc., vol. 1, Amer. Math. Soc., Providence, R. I., 1981, pp. 55-64.

2. , Knapp-Wallach Szegö integrals and P-induced continuous series representations: the parabolic rank one case, J. Funct. Anal. 60 (1985), 127-145.

3. Harish-Chandra, Discrete series for semisimple Lie groups. I, Acta Math. 113 (1965), 241-318.

4. Discrete series for semisimple Lie groups. II, Acta Math. 116 (1966), 1-111.

5. , On the theory of the Eisenstein integral, Lecture Notes in Math., vol. 266, Springer-Verlag, New York and Berlin, 1971, pp. 123-149.

6. Harmonic analysis on real reductive groups. I, J. Funct. Anal. 19 (1975), 104-204.

7. S. Helgason, Differential geometry and symmetric spaces, Academic Press, New York, 1962.

8. A. W. Knapp, A Szegö kernel for discrete series, Proc. Internat. Congress Math. 1974, vol. 2, Canad. Math. Congress, 1975, pp. 99-104.

9. , Commutativity of intertwining operators for semisimple groups, Compositio Math. 46 (1982), $33-84$.

10. A. W. Knapp and N. R. Wallach, Szegö kernels associated with discrete series, Invent. Math. 34 (1976), 163-200.

11. . Correction and addition to Szegö kernels associated with discrete series, Invent. Math. 62 (1980), 341-346.

12. A. W. Knapp and Gregg Zuckerman, Classification of irreducible tempered representations of semisimple groups, Ann. of Math. (2) 116 (1982), 389-455.

13. A. Koranyi and J. Wolf, Realization of Hermitian symmetric spaces as generalized half-planes, Ann. of Math. (2) 81 (1965), 265-288. 
14. G. W. Mackey, Induced representations of locally compact groups. I, Ann. of Math. (2) 55 (1952), 101-139.

15. W. Schmid, Homogeneous complex manifolds and representations of semisimple Lie groups, Thesis, Univ. of California, Berkeley, Calif., 1967.

16. On the realization of the discrete series of a semisimple Lie group, Rice Univ. Studies 56 (1970), 99-108.

17. On the characters of the discrete series (the Hermitian symmetric case), Invent. Math. 30 (1975), 47-144.

18. V. S. Varadarajan, Harmonic analysis on real reductive groups, Lecture Notes in Math., vol. 576, Springer-Verlag, New York, Berlin and Heidelberg, 1977.

Department of Mathematics, Washington University, St. Louis, Missouri 63130 